\title{
EFEITO DA APRESENTAÇÃO COMERCIAL DE ÁCIDO CLORÍDRICO PARA TÉCNICA DE MICROABRASÃO NA PERDA DE ESTRUTURA E SUPERFÍCIE DO ESMALTE
}

\author{
Effect of hydrochloric acid commercial presentation for microabrasion \\ technique on loss of enamel structure and surface
}

\author{
(D) Pâmela Lopes Pedro da Silvaa , (D) Patricia Pereira Maciela ${ }^{a}$, (D) Lídia Batista Conrado Martins ${ }^{b}$, (ID) Eliseu \\ Aldrigui Münchowc, (D) Rogério Lacerda-Santos ${ }^{d}$, (D) Fabíola Galbiatti de Carvalho ${ }^{d}$, (D) Hugo Lemes Carlo ${ }^{d}$
}

\section{RESUMO}

Introdução: A técnica de microabrasão pode ser realizada através de pasta pronta para uso, disponível comercialmente, ou o profissional pode manipulá-la no consultório. Objetivo: Verificar o efeito da apresentação comercial do ácido clorídrico a 10\% na manipulação de pasta para microabrasão sobre a superfície do esmalte. Metodologia: Foram selecionados incisivos bovinos e divididos em dois grupos, de acordo com a apresentação comercial do ácido clorídrico (líquido ou em gel). O tratamento foi realizado através de dez aplicações com 10s de duração cada, intercaladas por lavagem de 10s. Vinte incisivos $(n=10)$ foram utilizados para se determinar a perda de estrutura do esmalte. Cada amostra foi pesada, em balança analítica, antes e após submissão à microabrasão. Outras 20 amostras $(n=10)$ foram utilizadas para determinação da rugosidade superficial média (Ra) utilizando-se um rugosímetro. Três amostras de cada grupo do experimento anterior foram selecionadas, aleatoriamente, e outras três amostras adicionais foram preparadas como controle (baseline) para análise em MEV. Resultados: Verificou-se diferença estatística significativa entre a massa final e a inicial e rugosidade superficial das amostras, independente da apresentação comercial do ácido. Nas imagens de MEV observou-se presença de superfície regular para o grupo controle (baseline). Nas demais imagens verificou-se superfície com considerável irregularidade e dissolução discreta do esmalte. Conclusões: O tratamento realizado causou perda significativa de estrutura e aumentou a rugosidade superficial dos espécimes, independente da apresentação comercial do ácido e sem apresentar diferença entre os grupos ao final. A apresentação comercial do ácido não parece ser um fator a interferir no tratamento.

Palavras-chaves: Esmalte dentário. Microabrasão do esmalte. Desgaste dos dentes.

\section{ABSTRACT}

Introduction: The microabrasion technique can be performed using a commercially available paste, or the dentist can prepare it in his office. Objective: To verify the effect of hydrochloric acid commercial presentation in the handling of microabrasion paste on the enamel surface. Methodology: Bovine incisors were divided into two groups, according to the commercial presentation of $10 \%$ hydrochloric acid (liquid or gel). The treatment was carried out through ten applications of $10 \mathrm{~s}$ duration each, intercalated with a 10s wash. Twenty teeth $(n=10)$ were used to determine the loss of enamel structure. Each sample was weighed on an analytical balance before and after submission to microabrasion. Another 20 teeth $(n=10)$ were used to determine the average surface roughness ( $\mathrm{Ra}$ ) using a rugosimeter. Three samples from each group of the previous experiment were selected, randomly, and another three additional samples were prepared as a control (baseline) for SEM analysis. Results: There was a statistically significant difference between the final and initial mass and the surface roughness of the samples, regardless of the acid commercial presentation. In the SEM images, a regular surface was observed for the control group (baseline). In the other images, there was a surface with considerable irregularity and a slight dissolution of the enamel. Conclusions: The treatment carried out caused a significant loss of structure and increased the surface roughness of the specimens, regardless of the acid commercial presentation and without showing any difference between groups at the end of the treatment. The acid commercial presentation did not appear to be a factor interfering.

Keywords: Dental enamel. Enamel microabrasion. Tooth wear.

\footnotetext{
a Cirurgião-dentista. Programa de Pós-graduação em Odontologia. Universidade Federal da Paraíba, João Pessoa, PB, Brasil.

${ }^{\mathrm{b}}$ Cirurgião-dentista. Programa de Pós-graduação em Odontologia. Universidade Federal de Juiz de Fora, Juiz de Fora, MG, Brasil.

${ }^{\text {c }}$ Professor Adjunto. Departamento de Odontologia Conservadora. Faculdade de Odontologia. Universidade Federal do Rio Grande do Sul, Porto Alegre, RS, Brasil.

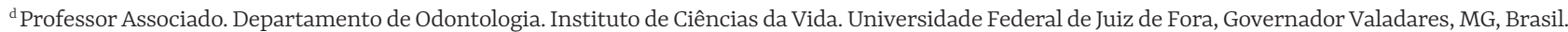

Autor de correspondência: Hugo Lemes Carlo - E-mail: hugo.carlo@ufjf.edu.br

Data de envio: 16/05/2020 | Data de aceite: 07/08/2020 


\section{INTRODUÇÃO}

Áreas brancas opacas ou com algum tipo de descoloração, apresentando uma camada superficial relativamente intacta ou até mesmo porosidades, podem resultar de algum distúrbio no processo de mineralização do esmalte ou de lesões de cárie causadas pelo ácido produzido durante a fermentação dos açúcares pelas bactérias do biofilme ${ }^{1-3}$. Estas alterações são tratadas, geralmente, através de três técnicas diferentes: remoção superficial do esmalte alterado, clareamento da estrutura dental e/ou recobrimento sem remoção ou com remoção parcial/total do tecido afetado. Tais técnicas correlacionam-se aos conceitos dos tratamentos de microabrasão do esmalte, clareamento de dentes vitais/não vitais e restaurações diretas ou indiretas. A escolha depende da gravidade da lesão ${ }^{2}$.

A microabrasão do esmalte trata-se de procedimento no qual uma pequena quantidade superficial do esmalte é simultaneamente erodida e abrasionada, deixando uma superfície de esmalte perfeitamente intacta ao final ${ }^{4,5}$. Este procedimento foi descrito pela primeira vez em 1986 por Croll e Cavanaughi ${ }^{i}$ descrevendo situações clínicas de tratamentos abrasivos bem-sucedidos empregando-se uma pasta composta de ácido clorídrico (HCl) a 18 \% associado ao pó de pedra-pomes de granulação fina. A quantidade de desgaste provocada depende da interação de diferentes fatores como, o tipo de ácido empregado, a quantidade de repetições realizadas e o tempo de aplicação ${ }^{5}$. Além disso, também se verificou que a concentração do ácido empregado e a pressão exercida pelo operador podem influir no resultado final do desgaste 6 .

A técnica proposta por Croll e Cavanaugh ${ }^{i}$ utilizando a pasta de ácido clorídrico e pedra pomes foi capaz de remover cerca de 25 a $200 \mu \mathrm{m}$ de esmalte, após 1 a 10 aplicações com duração de 1 min cada, quantidade esta que pode ser considerada irrisória com relação à quantidade de tecido remanescente $e^{7}$. O resultado final do tratamento resulta em um esmalte mais resistente à desmineralização bacteriana, diminuindo o risco de desmineralização progressiva ${ }^{8,9}$. A técnica apresenta-se como efetiva para recuperação da estética dental, principalmente por ser segura, rápida, simples e de resultado imediato, além de possuir baixo custo.

O uso de um abrasivo, como a pedra-pomes, não é suficiente para remover, sozinho, a superfície alterada do esmalte, sendo necessário o uso, também, do agente erosivo. Tem-se aí, a importância do uso de um ácido na técnica de microabrasão ${ }^{10}$. A princípio, a técnica preconizada recomendava o uso de ácido clorídrico na concentração de 18\%. Entretanto, sabe-se hoje que esta concentração pode ser tóxica e erosiva em excesso e, em decorrência disto, este ácido é utilizado em concentrações mais baixas, nos valores de 6,6\% e 10\% ${ }^{7,11}$. Além disso, verificou-se que o ácido fosfórico a $37 \%$ pode ser utilizado em substituição ao ácido clorídrico, apresentando resultados similares ${ }^{12}$. Podem ser utilizadas pastas prontas para o uso, disponíveis comercialmente, ou o profissional pode manipular sua própria pasta, a partir da mistura dos componentes, em seu consultório ${ }^{13-15}$.

Estudos foram realizados com o intuito de se mensurar a perda de estrutura, as possíveis alterações na morfologia do esmalte e o risco para a estrutura remanescente, relacionando-se com a rugosidade superficial após a realização do tratamento ${ }^{1,8,9,15-20}$. Contudo, não se verificou na literatura estudos que tenham quantificando, em massa, a perda de tecido após a realização da técnica. A pasta, quando manipulada, deve apresentar consistência adequada, evitando-se o extravasamento da mesma para áreas que não necessitam do tratamento, tornando o procedimento mais seguro e de mais fácil e rápida execução ${ }^{21}$. Este fator também foi pouco analisado pela literatura.

${ }^{i}$ Croll TP, Cavanaugh RR. Enamel color modification by controlled hydrochloric acid-pumice abrasion. I. Technique and examples. Quintessence Int. 1986 fev;17(6):81-7. 
O presente estudo teve como objetivo analisar o efeito, sobre o esmalte dental bovino, de pastas para microabrasão manipuladas com diferentes apresentações comerciais de ácido clorídrico a 10\% (líquida ou gel), caracterizando a perda de estrutura em massa (g) - através de análise gravimétrica, rugosidade superficial média (Ra) e topografia de superfície (MEV). A hipótese nula testada foi a de que a apresentação comercial do ácido não interferiria nos resultados obtidos.

\section{Material e métodos}

Foram utilizados 43 incisivos bovinos recém-extraídos, os quais foram raspados, com curetas de periodontia, e limpos, para retirada de tecidos moles, sendo posteriormente seccionados perpendicularmente ao seu longo eixo, para a remoção das raízes, utilizando-se disco de diamante (KG Sorensen, Barueri, Brasil) em peça de mão com refrigeração. Logo após foi retirada toda a polpa da câmara pulpar com limas endodônticas Hedströem (Maillefer, Ballaigues, Suiça). Os dentes passaram por um exame em lupa estereoscópica (aumento de 40x - STEMI SV11 - Zeiss, Oberkochen, Alemanha) para exclusão daqueles com trincas ou outras falhas em sua estrutura e então lavados em água corrente e acondicionados em água destilada a $4^{\circ} \mathrm{C}$, realizando-se a troca semanalmente até o momento do experimento.

O tratamento de microabrasão foi executado utilizando-se pasta a base de ácido clorídrico a 10\% manipulado em uma apresentação comercial mais fluida (na forma de um líquido) ou em uma apresentação comercial mais viscosa (na forma de um gel) (Farmácia Teixeira, João Pessoa, Brasil) e pó de pedra pomes (Quimidrol, Joinville, Brasil). Para padronização da manipulação das pastas, as mesmas foram preparadas na proporção de uma parte de pedra-pomes para uma parte do ácido, utilizando-se para isso a colher medida do cimento de ionômero de vidro Vidrion F (S.S.White, Rio de Janeiro, Brasil) ${ }^{20}$. Os materiais foram misturados, para a conformação da pasta, em um pote dappen e transferidos para a superfície das amostras utilizando-se uma seringa de $1 \mathrm{ml}$. Foram utilizados 0,04ml do material por aplicação.

A pasta abrasiva foi aplicada na superfície do esmalte em uma única sessão com um total de dez aplicações, utilizando-se taça de borracha (Microdont, São Paulo, Brasil) montada em micromotor e contra ângulo. Cada aplicação foi realizada pelo tempo de 10s e entre cada aplicação houve um intervalo de 10s para remoção da pasta de sobre a superfície das amostras utilizando-se jato de ar-água abundante. As taças de borracha foram trocadas a cada nova amostra. Todo o procedimento foi realizado por um único operador.

\section{Análise gravimétrica}

Os espécimes foram analisados quanto à perda de estrutura de esmalte utilizando-se análise gravimétrica ${ }^{22}$. Trata-se de avaliação indireta da eficiência de abrasão dos produtos testados, determinando-se a perda de massa do esmalte, em gramas (g). Para a realização desta análise foram utilizadas 20 (vinte) amostras, divididas em dois grupos experimentais $(\mathrm{n}=10)$ de acordo a apresentação comercial do ácido.

Cada amostra foi pesada em balança analítica (AG 200 - Gehaka, São Paulo, Brasil) com precisão de 0,00001g antes e após a realização do tratamento abrasivo, para determinação da quantidade de esmalte dental removido. Também foi calculada a porcentagem de massa perdida. Para padronização, antes de cada do procedimento de pesagem as amostras foram secas com papel absorvente, a qual foi seguida de secagem com jato de ar a $10 \mathrm{~cm}$ de distância por $40 \mathrm{~s}^{23}$. 


\section{Análise de rugosidade}

Para a realização desta análise também foram utilizadas 20 (vinte) amostras, divididas em dois grupos experimentais $(\mathrm{n}=10)$ conforme a apresentação comercial do ácido. As coroas dos dentes foram fixadas em tubos de PVC de $3 \mathrm{~cm}$ de altura utilizando-se resina acrílica, de modo que a face vestibular se mantivesse paralela à base do cilindro de PVC e ambos paralelos ao solo. Após a polimerização da resina acrílica, com a finalidade de padronização da superfície do esmalte, as mesmas foram polidas utilizando-se lixas d'água com granulação decrescente - lixa 320 (3M do Brasil, Sumaré, Brasil) até a superfície do dente tornar-se visivelmente plana; lixa 600 (3M do Brasil, Sumaré, Brasil) pelo período de 1min30s; e lixa 1200 (3M do Brasil, Sumaré, Brasil) pelo período de 1min30s - montadas em uma politriz (PSK-2V - Skill-tech, São Paulo, Brasil). Por fim, para isolar a área a ser trabalhada e promover impermeabilização, uma janela de $4 \mathrm{~cm}^{2}$ foi determinada utilizando-se esmalte para unhas (Risqué, São Paulo, Brasil) ${ }^{23}$.

Antes do teste de abrasão, as medidas de rugosidade superficial média (Ra) inicial foram realizadas em todos os espécimes (baseline). Os mesmos foram posicionados para medição em um rugosímetro (Surftest SJ 301 - Mitutoyo, Tóquio, Japão). O referido aparelho possui uma ponta de diamante de $2 \mu \mathrm{m}$ de raio, fazendo a leitura da superfície com velocidade constante de $0,05 \mathrm{~mm} / \mathrm{s}$ e carga de $0,7 \mathrm{~m} / \mathrm{N}$. O valor de cutoff foi fixado em $0,8 \mathrm{~mm}$ no filtro de Gauss. Três leituras foram realizadas em cada amostra para obtenção de uma média.

Após a abrasão, os espécimes foram lavados em banho de ultrassom (Ultrasonic Cleaner model USC1400 - Unique Ind e Com Ltda, São Paulo, Brasil) por 10min em água destilada e gentilmente secos com papel absorvente. Em seguida, três novas leituras foram realizadas em cada amostra, como descrito anteriormente, para obtenção da medida de rugosidade superficial média final.

\section{Microscopia Eletrônica de Varredura (MEV)}

Três amostras de cada grupo do experimento anterior foram selecionadas aleatoriamente para análise em MEV. Três amostras adicionais foram preparadas como controle (baseline) para o exame da topografia de superfície do esmalte após o polimento e sem a realização do procedimento de microabrasão. Após as amostras terem sido desidratadas em sílica em um recipiente a $40^{\circ} \mathrm{C}$ por três dias, as amostras foram montadas em stubbs de alumínio e revestidas com uma camada de ouro e platina (SCD-050 - Baltec, Balzers, Liechtenstein) e examinadas no Microscópio Eletrônico de Varredura (Leo 1430 - Zeiss, Oberkochen, Alemanha). As imagens foram obtidas com ampliação de 1.000x.

\section{Análise estatística}

Os dados das análises gravimétrica e de rugosidade obtidos foram submetidos a análise estatística através do Teste $t$ pareado $(\alpha=5 \%)$, a fim de se determinar se houve diferença significativa antes e após a realização do tratamento. Também foi realizado Teste $t(\mathbf{\alpha}=5 \%)$ para comparação dos resultados entre os grupos. 


\section{RESULTADOS}

O valor médio de massa, em gramas, dos espécimes antes e após serem submetidos à técnica de microabrasão, bem como o valor médio da perda de massa após a microabrasão, acompanhado do desvio padrão, estão apresentados na Tabela 1. Verificou-se diferença estatística significativa entre a massa final e a inicial das amostras, independente da apresentação comercial do ácido. Entretanto, não se verificou diferença estatística significativa com relação à perda de massa (\%), após a microabrasão, tanto para o ácido em sua consistência líquida $(1,12 \pm 0,51)$ quanto para o ácido na consistência em gel $(1,28 \pm 0,43)$.

Tabela 1: Resultados de massa das amostras (g) antes e após os espécimes serem submetidos à microabrasão e de perda de massa (\%) após o tratamento.

\begin{tabular}{llll}
\hline $\begin{array}{l}\text { Apresentação comercial } \\
\text { do ácido clorídrico }\end{array}$ & $\begin{array}{l}\text { Massa inicial }(\mathbf{g}) \\
(\text { Média } \pm \text { DP) }\end{array}$ & $\begin{array}{l}\text { Massa final }(\mathbf{g}) \\
(\text { Média } \pm \text { DP) }\end{array}$ & $\begin{array}{l}\text { Perda de massa }(\%) \\
(\text { Média } \pm D P)\end{array}$ \\
\hline Líquido & $3,675( \pm 0,523)^{\mathrm{A}}$ & $3,634( \pm 0,524)^{\mathrm{B}}$ & $1,28( \pm 0,43)^{\mathrm{a}}$ \\
Gel & $4,062( \pm 0,759)^{\mathrm{A}}$ & $4,010( \pm 0,745)^{\mathrm{B}}$ & $1,12( \pm 0,51)^{\mathrm{a}}$ \\
\hline
\end{tabular}

Letras maiúsculas diferentes indicam diferença estatística significativa entre a massa inicial e final das amostras após a realização do tratamento. Teste $t$ pareado $(p<0,05)$.

Letras minúsculas iguais indicam não haver diferença significativa para perda de massa entre os grupos testados. Teste $t(p>0,05)$.

O valor médio da rugosidade superficial do esmalte antes e após técnica de microabrasão, bem como o desvio-padrão para cada um dos grupos experimentais, estão apresentados na Tabela 2. Observou-se aumento significativo $(\mathrm{p}<0,05)$ nos resultados de rugosidade superficial, independente da apresentação comercial do ácido empregado, após a realização da abrasão. Entretanto, não se observou diferença significativa $(\mathrm{p}>0,05)$ quando se compararam os grupos antes $(0,21 \pm 0,02 / 0,21 \pm 0,01)$ ou após $(0,56 \pm 0,11 / 0,65 \pm 0,09)$ a microabrasão.

Tabela 2: Resultados de rugosidade superficial média ( $\mu \mathrm{m})$ e desvio-padrão antes e após microabrasão.

\begin{tabular}{|c|c|c|}
\hline $\begin{array}{l}\text { Apresentação comercial do } \\
\text { ácido clorídrico }\end{array}$ & $\begin{array}{l}\text { Rugosidade Inicial } \\
\text { Média ( } \pm D P \text { ) }\end{array}$ & $\begin{array}{l}\text { Rugosidade Final } \\
\text { Média ( } \pm \text { DP) }\end{array}$ \\
\hline Líquido & $0,21( \pm 0,02) \mathrm{Aa}$ & $0,56( \pm 0,11) \mathrm{Bb}$ \\
\hline Gel & $0,21( \pm 0,01) \mathrm{Aa}$ & $0,65( \pm 0,09) \mathrm{Bb}$ \\
\hline
\end{tabular}

Letras maiúsculas diferentes indicam diferença estatística significativa entre a rugosidade superficial inicial e final das amostras após a realização do tratamento. Teste t pareado $(p<0,05)$.

Letras minúsculas iguais indicam não haver diferença significativa para rugosidade superficial entre os grupos testados antes ou após a realização do tratamento. Teste $t(p>0,05)$.

Ao se analisar as imagens obtidas através da Microscopia Eletrônica de Varredura observou-se uma superfície mais lisa na Figura 1a - Controle (Baseline), com a presença de pequenas ranhuras advindas do processo de polimento das amostras. Nas demais imagens (Figuras 1b e 1c - ácido clorídrico nas apresentações comerciais gel e líquido, respectivamente) verificou-se uma superfície com considerável irregularidade e dissolução discreta do esmalte. 


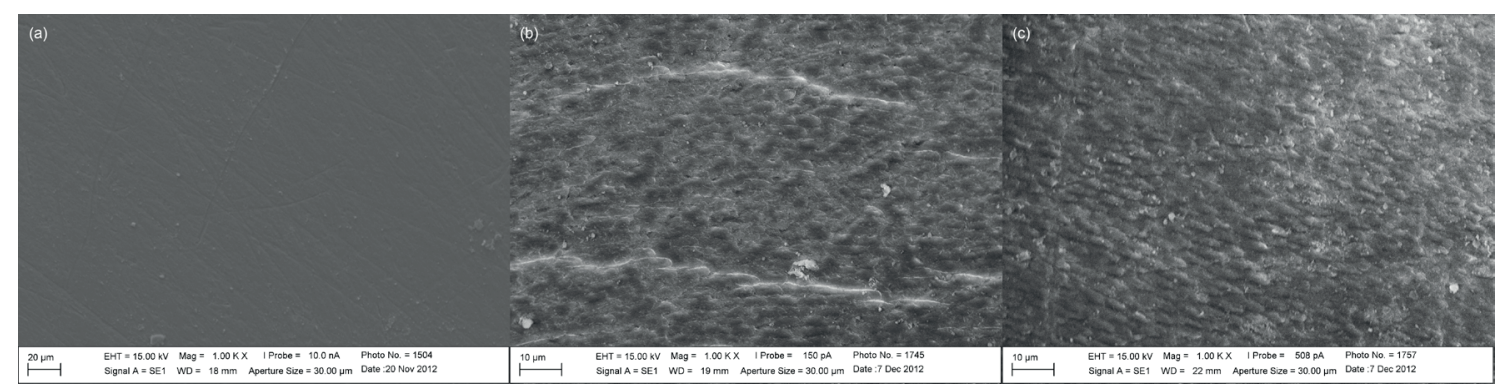

Figura 1: Imagens de Microscopia Eletrônica de Varredura da superfície do esmalte: (a) Controle (Baseline); (b) após tratamento de microabrasão com pasta de ácido clorídrico na viscosidade de gel; e (c) após tratamento com pasta de ácido clorídrico na viscosidade líquida.

\section{DIscussão}

Este estudo verificou o efeito de pastas para microabrasão manipuladas com diferentes apresentações comerciais de ácido clorídrico a 10\% (líquida ou gel) sobre o esmalte dental bovino. Verificou-se diferença estatística significativa entre as massas inicial e final, independente da apresentação comercial, não se verificando diferença significativa dos resultados entre os ácidos testados. O mesmo resultado foi observado na análise de rugosidade superficial. Desta forma, a hipótese nula testada foi aceita, pois a apresentação comercial do ácido não interferiu nos resultados obtidos.

A literatura demonstra que a microabrasão do esmalte é o método mais rápido e fácil para a remoção de manchas dentais superficiais. No entanto, cuidados devem ser tomados durante a execução da técnica, pois o material ácido empregado tem efeito cáustico, o que pode causar danos ao paciente ou à equipe profissional. Além disso, remoção excessiva de estrutura dentária pode ocorrer caso a técnica não seja bem executada ${ }^{24}$.

As amostras foram analisadas por meio de análises gravimétrica e rugosimétrica. A análise gravimétrica se deu conforme Tavares da Silva et al. ${ }^{23}$, que avaliaram a eficiência de desgaste de pontas diamantadas, por meio da pesagem, em dentes humanos. $O$ teste compreende uma avaliação indireta da eficiência de abrasão dos produtos testados sobre o esmalte, por meio da perda de massa do substrato de desgaste. Os resultados desta análise demonstraram que, independente da apresentação comercial do ácido, a técnica de microabrasão provocou uma diminuição significativa na massa dos espécimes analisados, ou seja, causou perda da estrutura do esmalte do elemento dental, sendo que não se observou diferença estatística significativa entre os grupos. Frente à ausência de outros estudos semelhantes e a falta de literatura para comparação não se pode afirmar que essa quantidade de tecido removido ocorreu em demasia, mas pode-se supor que sim, pois pastas comerciais para microabrasão contendo $\mathrm{HCl}$ a $6 \%$ e carboneto de silício apresentaram resultados clínicos aceitáveis ${ }^{20}$, com a perda de esmalte variando de 10 a $200 \mu \mathrm{m}$ na superfície do esmalte ${ }^{2}$. Este tipo de desgaste é muito menor do que aquele verificado quando da utilização de pontas diamantadas ${ }^{23}$.

A análise de rugosidade trata-se de um método capaz de avaliar discretas alterações causadas pelo tratamento à superfície do esmalte ${ }^{25}$. Os efeitos da microabrasão estão relacionados aos componentes erosivos (ácidos) e abrasivos (pedra-pomes ou sílica) da pasta, onde os abrasivos desempenham papel relevante ${ }^{10}$, permitindo maior atrito na superfície e resultando em uma camada superficial de esmalte mais rugosa ${ }^{11}$. $\mathrm{O}$ agente abrasivo usado neste estudo foi a pedra-pomes, considerado como aquele de maior abrasividade e com erosão mais profunda quando comparado à sílica, por exemplo ${ }^{20}$. Um maior aumento de rugosidade foi observado no esmalte quando do uso de pedra-pomes em comparação com 
pastas contendo partículas de sílica, possivelmente devido à presença de grãos com menor tamanho e distribuição mais uniforme $e^{16,20,26,27}$.

De acordo com estudos anteriores ${ }^{1,15,16,19}$, o uso de ácido clorídrico em diferentes concentrações alterou a rugosidade das amostras submetidas ao processo microabrasão, podendo ser justificado pelo poder erosivo do ácido, resultado de um condicionamento não seletivo e ausência de padronização da demineralização da superfície dentária ${ }^{1}$. Neste estudo, todas as técnicas microabrasivas apresentaram aumento nos índices de rugosidade superficial, o que já foi previamente relatado ${ }^{28}$, porém sem diferença estatística significativa entre os materiais utilizados. O polimento realizado nas amostras do grupo baseline se apresentou bastante reprodutível, visto que não se observou resultado com diferença significativa entre as mesmas.

A superfície do esmalte foi alterada pela realização do tratamento de microabrasão. As imagens de MEV apresentam a superfície do esmalte sem tratamento (baseline) e as alterações causadas pelo ácido. Observa-se uma superfície visivelmente mais lisa na amostra representativa do grupo baseline (Figura 1a), com a presença de pequena quantidade de ranhuras advindas do processo de polimento realizado. Observa-se nas outras imagens (Figuras 1b e 1c) a presença de grande quantidade de irregularidades oriundas da dissolução discreta da superfície do esmalte. Alterações morfológicas semelhantes já foram verificadas ${ }^{13,19,26}$.

A ocorrência de desmineralização erosiva normalmente se mantém restrita à superfície do esmalte até que ocorra uma perda significativa de tecido na região. O ácido atingirá as camadas mais profundas, somente, após a efetivação de sua ação na superfície, aumentando os poros do esmalte e facilitando sua penetração. Assim, verifica-se que a apresentação comercial do ácido tem a capacidade de retardar sua penetração nas camadas mais profundas, a não ser que sua ação seja potencializada pela técnica de aplicação ${ }^{17}$. Esta, provavelmente, trata-se da justificativa para que a perda de massa e a rugosidade não tenham sido influenciadas pela diferença de apresentações comerciais, pois a aplicação das pastas foi realizada em ambos os grupos, de forma semelhante a outros estudos, com o uso de instrumento rotatório (taça de borracha) ${ }^{18,27,29}$. Entretanto, deve-se salientar que tal técnica pode ter potencializado a perda de tecido, pois já foi demonstrado que a microabrasão, quando realizada através de técnica manual de aplicação, apresenta menor desgaste da estrutura dental ${ }^{18,29}$.

As pastas de ácido clorídrico foram manipuladas conforme descrito anteriormente ${ }^{4}$, onde o ácido é misturado ao abrasivo (normalmente pedra-pomes) na proporção de 1:1 em volume. A mistura dos dois materiais forma uma pasta com uma consistência que facilita a aplicação e manipulação sobre a superfície do esmalte, colaborando para um menor escoamento da pasta durante o uso do instrumento rotatório ${ }^{6}$. A pasta manipulada com o ácido clorídrico na condição líquida apresentou uma consistência mais fluida, o que dificultou o controle da pasta sobre a superfície do esmalte, diferentemente do material na consistência de gel, que permitiu um melhor controle de aplicação da pasta e resultou em maior segurança para execução da técnica. Não se aumentou a quantidade de abrasivo para compensação da fluidez da pasta, pois uma maior quantidade de abrasivo poderia acarretar maior rugosidade e maior perda de estrutura ${ }^{27}$. Desta forma, sugerimos que o uso do agente erosivo na consistência de gel deveria ser preferido em relação ao seu homólogo na consistência mais fluida.

A duração e a quantidade das aplicações, por sessão, ainda não foram completamente estabelecidas na literatura ${ }^{1,12,22,30-32}$. Alguns estudos preconizaram a execução de dez aplicações com duração de 10 sada $^{13,19}$. Já outros sugeriram que a sessão deveria ser realizada com cinco aplicações sem o estabelecimento de tempo mínimo ou máximo ${ }^{33,34}$. O presente estudo fez a opção por dez aplicações com 10s de duração como limite máximo, por ser o mais corriqueiro na literatura ${ }^{16,19}$.

As pastas foram manipuladas utilizando-se ácido clorídrico na concentração de $10 \%$. O material já foi utilizado na concentração de $18 \%^{11,25}$ e os resultados de tais estudos diferem 
do atual provavelmente devido a esta diferença na concentração do mesmo. Entende-se hoje que o uso de ácidos com concentrações mais baixas, menos tóxicos ${ }^{4}$, podem de minimizar as injurias causadas pelo produto ${ }^{7,32}$.

A microabrasão é descrita como um método pouco invasivo por provocar, apenas, alterações micromorfológicas na superfície do esmalte. Tais alterações tornam o esmalte mais rugoso e poroso devido à capacidade do ácido em deslocar íons. Técnica de polimento deve ser realizada após o procedimento, seguido de aplicação tópica de flúor, pois tais procedimentos serão fundamentais para minimizar as alterações causadas, além de melhorarem o resultado estético obtido ${ }^{10,17,29}$.

A técnica de microabrasão é um procedimento, dentre outros, indicado para o tratamento de áreas brancas opacas ou com algum tipo de descoloração no esmalte, sendo considerada como o tratamento de primeira escolha nestas situações ${ }^{21,35,36}$. Deve ser realizada com cautela para evitar remoção excessiva de estrutura sadia. E a apresentação comercial do ácido não deve ser um fator a interferir no resultado final. Quando se trabalhando com uma pasta mais fluida, cuidados devem ser tomados no sentido de se controlar sua aplicação sobre a superfície a ser tratada. Além disso, reitera-se o cuidado com a execução da técnica.

\section{CONCLUSÕES}

Com os achados deste estudo concluiu-se que:

- O tratamento de microabrasão do esmalte realizado causou perda significativa de estrutura em massa, e aumentou a rugosidade superficial média dos espécimes, independente da apresentação comercial do ácido e sem apresentar, ao final, diferença entre os grupos;

- A apresentação comercial do ácido não deve ser um fator a interferir no resultado do tratamento executado.

\section{REFERÊNCIAS}

1. Bertoldo C, Lima D, Fragoso L, Ambrosano G, Aguiar F, Lovadino ]. Evaluation of the effect of different methods of microabrasion and polishing on surface roughness of dental enamel. Indian ] Dent Res. 2014 mai/jun;25(3):290-3.

2. Bharath KP, Subba Reddy VV, Poornima P, Revathy V, Kambalimath HV, Karthik B. Comparison of relative efficacy of two techniques of enamel stain removal on fluorosed teeth. An in vivo study. J Clin Pediatri Dent. 2014;38(3):207-13.

3. Honorio HM, Rios D, Santos CF, Magalhaes AC, Delbem AC, BuzalafMA, etal. Cross-sectional microhardness of human enamel subjected to erosive, cariogenic or combined erosive/cariogenic challenges. Carie Res. 2010;44(1):29-32.

4. Mondelli ], Mondelli R, Bastos MTAA, Franco E. Microabrasão com ácido fosfórico. Rev Bras Odontol.1995;3(52):20-2.

5. Andrade FB, Gomes M]. Microabrasão: um recurso para a recuperação da estética dental. Odontologia Clín-Científ. 2007 jan/mar;1(6):9-25.

6. Olin PS, Lehner CR, Hilton JA. Enamel surface modification in vitro using hydrochloric acid pumice: an SEM investigation. Quintessence Int.1988 out;19(10):733-6.

7. Sundfeld RH, Croll TP, Briso AL, de Alexandre RS, Sundfeld Neto D. Considerations about enamel microabrasion after 18 years. Am ] Dent. 2007 abr;20(2):67-72.

8. Donly K], Sasa IS. Potential remineralization of postorthodontic demineralized enamel and the use of enamel microabrasion and bleaching for esthetics. Semin Orthod. 2008;14:220-5.

9. Lynch CD, McConnell R]. The use of microabrasion to remove discolored enamel: a clinical report. ] Prosthet Dent. 2003;90(5):417-9.

10. Paic M, Sener B, Schug ], Schmidlin Pr. Effects of microabrasion on substance loss, surface roughness, and colorimetric changes on enamel in vitro. Quintessence Int. 2008 jun;39(6):517-22. 
11. Murphy TC, Willmot DR, Rodd HD. Management of postorthodontic demineralized white lesions with microabrasion: a quantitative assessment. Am J Orthod Dentofacial Orthop. 2007 jan;131 (1):27-33.

12. Bassir MM, Bagheri G. Comparison between phosphoric acid and hydrochloric acid in microabrasion technique for the treatment of dental fluorosis. J Conserv Dent. 2013 jan;16(1):41-4.

13. Pini NI, Lima DA, Ambrosano GM, da Silva W], Aguiar FH, Lovadino JR. Effects of acids used in the microabrasion technique: microhardness and confocal microscopy analysis. J Clin Exp Dent. 2015 out;7(4):e506-12.

14. Tagliari D, Kirchhoff AL, Grando KG, Reis A, Joaçaba SC. Análise da rugosidade do esmalte bovino submetido a microabrasão com diferentes materiais. Rev Ibero-am Odontol Estet Dent. 2003;2(8):337-46.

15. Yazkan B, Ermis RB. Effect of resin infiltration and microabrasion on the microhardness, surface roughness and morphology of incipient carious lesions. Acta Odontol Scand. 2018 out;76(7):473-81.

16. Fragoso LS, Lima DA, de Alexandre RS, Bertoldo CE, Aguiar CE, Lovadino JR. Evaluation of physical properties of enamel after microabrasion, polishing, and storage in artificial saliva. Biomed Mater. 2011 jun; 6(3):035001.

17. Mathias ], Kavitha S, Mahalaxmi S. A comparison of surface roughness after micro abrasion of enamel with and without using CPP-ACP: an in vitro study. J Conserv Dent. 2009 jan;12(1):22-5.

18. Nevárez-Rascón M, Molina-Frechero N, Adame E, Almeida E, Soto-Barreras U, Gaona E, et al. Effectiveness of a microabrasion technique using $16 \% \mathrm{HCL}$ with manual application on fluorotic teeth: a series of studies. World J Clin Cases. 2020;8(4):743-56.

19. Pini NI, Costa R, Bertoldo CE, Aguiar FH, Lovadino JR, Lima DA. Enamel morphology after microabrasion with experimental compounds. Contemp Clin Dent. 2015 abri/jun;6(2):170-5.

20. Rodrigues MC, Mondelli RF, Oliveira GU, Franco EB, Baseggio W, Wang L. Minimal alterations on the enamel surface by micro-abrasion: in vitro roughness and wear assessments. J Appl Oral Sci. 2013 mar/abri;21(2):112-7.

21. Croll TP. Enamel microabrasion: the technique. Quintessence Int. 1989 jun;20(6):395-400.

22. Souza de Barros Vasconcelos MQ, Almeida Vieira K, da Consolação Canuto Salgueiro M, Almeida Alfaya T, Santos Ferreira C, Bussadori SK. Microabrasion: a treatment option for white spots. J Clin Pediatr Dent. 2014;39(1):27-9.

23. Tavares da Silva RHB, Miranda AF, Pimenta FB, Pita APG, Pinelli LAP. Efeito da desinfecção química sobre a eficiência de corte de pontas diamantadas. Rev Odontol UNESP. 2002;2(31):259-68.

24. Castro KS, Ferreira AC, Duarte RM, Sampaio FC, Meireless SS. Acceptability, efficacy and safety of two treatment protocols for dental fluorosis: a randomized clinical trial. J Dent. 2014 ago;42(8):938-44.

25. Meireles SS, Andre Dde A, Leida FL, Bocangel JS, Dermaco FF. Surface roughness and enamel loss with two microabrasion techniques. J Contemp Dent Pract. 2009 jan;10(1):58-65.

26. Pini Nip, Sundfeld-Neto D, Aguiar Fhb, Sundfeld RH, Martins LRM, Lovadino]R. Microabrasão de esmalte: uma visão geral de considerações clínicas e científicas. Casos do mundo. J Clin. 2015;16(1):34-41.

27. Tong LS, Pang MK, Mok NY, King NM, Wei SH. The effects of etching, micro-abrasion, and bleaching on surface enamel. J Dent Res. 1993 jan;72(1):67-71.

28. Bertoldo CE, Pini NI, Miranda DA, Catelan A, Ambrosano GM, Lima DA, et al. Physicochemical properties of enamel after microabrasion technique. ] Res Dent. 2014;2(2):176-88.

29. Ramos C], Judica C, Silvio Issao M, Keiko S, Chavez A, Elias V. Efeitos da microabrasão sobre mancha branca de cárie inativa em dentes decíduos. Pesq Bras Odontoped Clin Integr. 2006 mai/ago;2(6):149-54.

30. Balan B, Madanda Uthaiah C, Narayanan S, Mookalamada Monnappa P. Microabrasion: an effective method for improvement of esthetics in dentistry. Case Rep Dent. 2013;951589.

31. Celik EU, Yildiz G, Yazkan B. Clınıcal evaluatıon of enamel mıcroabrasıon for the aesthetıc management of mıld-to-severe dental fluorosis. ] Esthet Restor Dent. 2013 set/dez;25(6);422-30.

32. Sundfeld RH, Sunfeld-Neto D, Machado LS, Franco LM, Fagundes TC, Briso AL. Microabrasion in tooth enamel discoloration defects: three cases with long-term follow-ups. ] Appl Oral Sci. 2014 jul/agos;22(4):347-54.

33. De Melo RM, Travassos AC, Neisser MP. Shear bond strengths of a ceramic system to alternative metal alloys. J Prosthet Dent. 2005 jan;93(1):64-9.

34. Sheoran N, Garg S, Damle SG, Dhindsa A, Opal S, Gusta S. Esthetic management of developmental enamel opacities in young permanent maxillary incisors with two microabrasion techniques — a split mouth study. ] Esthet Restor Dent. 2014 set/out;26(5):345-52.

35. Croll TP, Cavanaugh RR. Enamel color modification by controlled hydrochloric acid-pumice abrasion. I. Technique and examples. Quintessence Int. $1986 \mathrm{fev} ; 17(6): 81-7$.

36. Croll TP, Cavanaugh RR. Enamel color modification by controlled hydrochloric acid-pumice abrasion. II. Further examples. Quintessence Int. 1986 Mar;17(3):157-64. 